\title{
MULTIPAYLOAD INJECTION CONCEPTION
}

\author{
G. Martens ${ }^{1}$, O. Lukyanovych ${ }^{2}$, and I. Filippenko ${ }^{3}$ \\ ${ }^{1}$ Colleferro, Italy \\ ${ }^{2}$ RPE HARTRON-ARKOS \\ Kharkov, Ukraine \\ ${ }^{3}$ SDO "Yuzhnoye" \\ Dniepropetrovsk, Ukraine
}

\begin{abstract}
The proposed approach is based on the decomposition of the orbital phase timeline into the closed "standard" phases - basic activities. Assembling the basic activities of flight timeline in accordance with multipayload release logic, there can be built the orbital phase of payload (PL) pointing of any degree of complexity. The method of additional flight timeline allows injection of any number of spacecrafts to the different Low Earth Orbits (LEO) assuming their feasibility by launch vehicle (LV) energetic performances. The important point of this approach is that the assembling of the orbital phase of a flight is provided by tuning of the mission data flags without change of the onboard software (SW) source code.
\end{abstract}

\section{STATEMENT OF PROBLEM}

Actually, the cost of PL injection to the LEO becomes an important part and has a significant impact on the design of mission strategy. One of the most efficient ways to reduce the injection cost is a multipayload mission, i. e., the injection of a group of the spacecrafts to one or few LEOs by the same launcher.

At present, there is no common (unified) conception of multipayload release. Every time, for every mission, this problem is being solved individually, without respect to the experience of previous (or parallel) solutions. This leads to considerable costs for developing, modification, and testing of the GNC (Guidance, Navigation, and Control systems) algorithms and onboard SW with respect to requirements of customer. Evidently, the development of the modern unified conception of multipayload release is necessary.

This paper performs an attempt to propose a multipayload release conception that can be considered as unification try. It allows an injection of any number 
of spacecrafts to different LEOs assuming their feasibility by LV energetic performances.

\section{BASIC PRINCIPLES OF THE CONCEPTION}

\subsection{Feasible Cases of Payload's Assembly and Separation}

Let describe briefly the feasible cases of multipayload's assembly, i. e., a group of two or more spacecrafts on one launcher. From this point of view, there are the following cases of mounting:

- several large-size satellites (monoblocks); and

- several large-size satellites (monoblocks) and a group of microsatellites.

The most attractive case by cost/functionality criterion is one or several large-size satellites and a group of microsatellites.

When the mounting of the PLs is being specified, the following characteristics should be accounted for:

- the number and weight of PLs;

- the case of PL mounting on the launcher; and

- the separation priority.

The PL mounting is one of the most important characteristic influencing the multipayload injection logic. For this purpose, different adapters and dispensers are used.

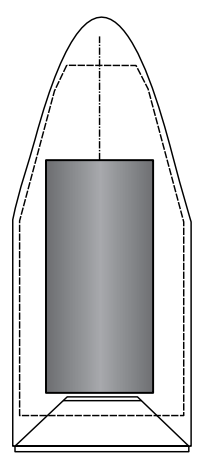

(a)

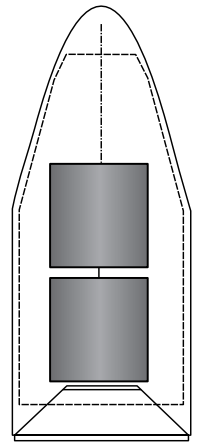

(b)

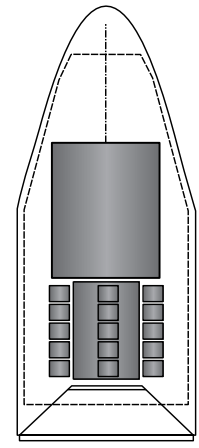

(c)

Figure 1 Payload mounting cases 
The following important point is the priority of the PL separation. Usually, the large-size satellites are separated earlier than microsatellites. This approach is quite justified from both scientific and financial points of view because large-size satellites define the main target of the mission. If satellites have equal priority, the separation sequence is determined by the spacecrafts mounting place/geometry.

Some cases of the PL's mounting are shown in Fig. 1.

Generally speaking, the approach proposed here allows the synthesis of the separation phase to any cases of arrangement.

\subsection{Main Concepts of Multipayload Injection}

One of the most important points of the theoretical methodology design is terminology unification. It provides clear and common language to describe the main statements of the approach.

The following concepts are introduced:

- insertion phase is the phase of flight starting from the GNC activation up to the first upper stage Main Engine (ME) cutoff;

- orbital phase is the phase of flight starting from the first upper stage ME cutoff up to the GNC deactivation;

- separation phase is the subphase of the orbital phase dedicated to the PL separation. It is activated by the "Spacecraft separation" command and completed by distancing from the released PL. This phase includes a full set of maneuvers required by the PL;

- flight operation - this term is used to define some elementary action performed in order to modify the launcher state, for example, the rotation around the axis of Body-fixed reference frame or the ME ignition/cutoff;

- basic activity is the flight operation or the sequence of flight operations which are unified by a common objective. A sequence of the basic activities forms full flight timeline of the orbital phase. The start and the end of the basic activity are determined by functional commands and labels of the Control system;

- attitude pointing maneuver is the flight operation changing the launcher attitude;

- additional ME ignition is the flight operation to ignite the upper stage ME in order to supply delta velocity and to modify the orbit required by the PL or by the mission evolution; 
- spin is the flight operation that provides the rotation of the launcher around the longitudinal axis before PL separation if the rotation is required by PL;

- PL separation is the flight operation indicating a physical process of the PL separation from the launcher. Implementation of this process depends on the case of PL arrangement; and

- mission scheme - here, it is the sequence of the basic activities that provides an insertion of the PL group to the required orbits with required attitude.

\subsection{Specification of the Basic Activities}

Full set of flight operations necessary to be implemented for PL injection at the orbital phase is defined by spacecraft functionality requirements. Let reunite the separated flight operations in some logical sequence.

Attitude pointing maneuver is applied for orientation axes of Body-fixed reference frame to the required position before PL separation. The aim of this step is ensuring of required orientation of launcher before PL separation. The maneuver could be implemented by one spatial turn simultaneously around three axes of Body-fixed reference frame or by consecutive turns around specified axes of Body-fixed reference frame. The required orientation is specified in the reference frame associated with the target orbit.

Spin before PL separation is implemented by using a low-thrust propulsion system.

Separation of PL from the launcher is the sequence of flight operations of large-size satellites or group of microsatellites separation.

Despin after PL separation is implemented by applying of sign opposite spin torque (angular rate in roll channel damping).

Contamination/collision avoidance maneuver is performed for the orientation of the launcher to the required attitude after PL separation. The aim of this maneuver is to avoid the PL contamination or collision with launcher after PL separation before withdrawal of the launcher from a spacecraft. The maneuver could be implemented by one three-dimensional (3D) turn simultaneously around three axes of Body-fixed reference frame or by consecutive turns around specified axes of Body-fixed reference frame. Required orientation is specified in the reference frame associated with the target orbit.

Withdrawal is an additional ignition of the engine. This basic activity is applied for withdrawal of the launcher from spacecraft in order to avoid the PL contamination or collision with the launcher. Main engine or low-thrust propulsion could be used.

Attitude pointing maneuver before transferring to an other orbit is performed for the orientation the axes of Body-fixed reference frame to the required attitude 
before transferring to other orbit. The aim of this maneuver is ensuring of the required launcher attitude before additional ME ignition. The maneuver could be implemented by one 3D turn simultaneously around three axes of Bodyfixed reference frame or by consecutive turns around specified axes of Body-fixed reference frame. Required orientation is specified in reference frame associated with target orbit.

Transferring to another orbit is additional ignition of the engine. This basic activity is applied for transferring the launcher to another orbit. For transferring, ME or low-thrust propulsion system could be used.

Long coasting phase is a phase of the flight between two consequent $\mathrm{ME}$ ignitions. This basic activity is performed for the implementation of the Homan orbit transfer. Fixed orientation (for example, for telemetry stations link) or slow rotation (to avoid local heating) could be required.

Every basic activity is defined by special commands and labels generated once some functional reaches the predefined threshold. Here, it is important to note that every basic activity could include all flight operations (attitude pointing maneuver, ME ignition, etc.) required for multipayloads injection. Finally, the integration degree is determined by the reasons of clarity and logicality of orbital phase formation.

Using the abovementioned set of basic activities and combining them according to the requirements accepted for a mission, it is possible to synthesize practically any orbital phase flight timeline.

\subsection{Principles of Orbital Phase Flight Timeline Synthesis}

The synthesis of the multipayloads injection logic is based on the following principles:

(1) effectiveness;

(2) safety;

(3) functionality; and

(4) environmental safety. tion.

Let discuss the every points concerning the problem of multipayloads injec-

Effectiveness is related closely with economical and technical reasonability. Evidently, multipayloads injection will be the most widespread mode of (outer) space development. It is connected with the tendency of modern spacecrafts mass and size decreasing. Therefore, all researches, which have the aim to find the system solution in this field of science, have not only scientific interest but also a particular economical sense. 
Safety here is ensuring of no damage of the satellites by the launcher structure, ME blast, combustion gas of the satellites separated earlier. Depending on case of arrangement, there are few approaches that could be used for the referred requirement implementation.

If the satellite is separated along the launcher longitudinal axis, the following flight operations are performed: orientation of the launcher's longitudinal axis toward the required direction before PL separation, PL release, reorientation of the launcher longitudinal axis in a safe direction after the satellite separation, withdrawal of the launcher by the ME or low-thrust propulsion system. In the case of multipayloads injection, all the set of flight operations is performed for every satellite. Such complex of activities ensures the execution of all safety requirements.

Therewith, a different way of separation could be used - along the line perpendicular to the long axis of the launcher. For example, it is used when a group of microsatellites is separated. That could be implemented by separation of couples of microsatellites in an opposite direction at the same time. This approach is more attractive for the flight algorithms complexity point of view, as well as for the fuel consumption. But this approach requires careful analysis of safety ensuring.

Functionality here is a possibility to generate the flight timeline of any level of complexity by using only GNC capacity. In this statement, the number of satellites and orbits is not constrained by GNC but only by the launcher energetic capacity. The orbital phase timeline is generated by using a combination of basic activities. Then orbital phase could be tuned only by the mission data. The proposed approach has considerable advantage comparing with the widely used methods as it allows to prepare SW for any mission by using the most simple and convenient way - changing constants of the flight mission data without changing the onboard SW sources code.

Nowadays, a great attention is paid to the space debris increasing and ecological safety of the orbital operations. One of the important points is limitation of the space debris remaining in the orbit. Therefore, deorbiting of the launcher upper stage is mandatory for multipayload injection.

\section{MULTIPAYLOAD INJECTION LOGIC}

\subsection{Multipayload Injection Logic Realization in General Formulation}

Multipurpose system is one of the most attractive ways of launchers evolution. This system should ensure implementation of all known functionality requirements. The potential evolution of these requirements also should be forestalled. 
However, conflicts between requirements, technical capacities, and constraints have significant influence on the characteristics of the system. Development of the multipayload injection logic and using high-performance onboard computer which allows to implement the algorithms of high level of complexity without any damages for flight safety is one of the resolutions of such conflicts. In this case, the possibility of separation of any number of satellites at any (physically justified) moments of the orbital phase of flight is being discussed.

Usually, this problem is solved by the development of the "hard-coded" timeline for each mission. Such timeline realizes sequentially all necessary basic activities and foresees a separation of all PLs at the required moments. In this case, it provides the implementation of all mission requirements. However, this approach has a grave shortcoming - if the number of PLs that are separated at every orbit is changed, it is necessary to remake all the timeline in accordance with new requirements, not mentioning changing the set or the sequence of basic activities. Therefore, this approach could be used only for solution of a particular problem of multipayloads injection and is unacceptable for mission strategic planning.

Another approach of flight timeline generation is based on the multiple using of the "standard" legs of flight including legs of PL's separation. Each leg consists of a fixed set of basic activities and has its own identifier. Generating the required sequence of the identifier, flight timeline is defined according to the mission scheme. Despite evident advantages of this approach, there are also a number of inconveniences in the usage of it. One of them is some uncertainty (functional commands and labels in this realization are not defined clearly) that could make a source of errors. Also, the process of the flight timeline logic generation is quite complex. At the phase of the mission preparation, the developers deal with fully modified onboard software. It complicates the GNC system testing process.

In consideration of all the abovementioned, the method of additional timeline for multipayloads logic realization has been designed. It is based on the idea of using two parallel independent timeline:

(1) main flight timeline; and

(2) additional PL's separation timeline.

Main timeline is divided into two phases:

(1) the insertion phase; and

(2) the orbital phase.

Insertion phase timeline represents the set and sequence of basic activities necessary for the mission scheme realization in this phase. It is unified for all missions. 


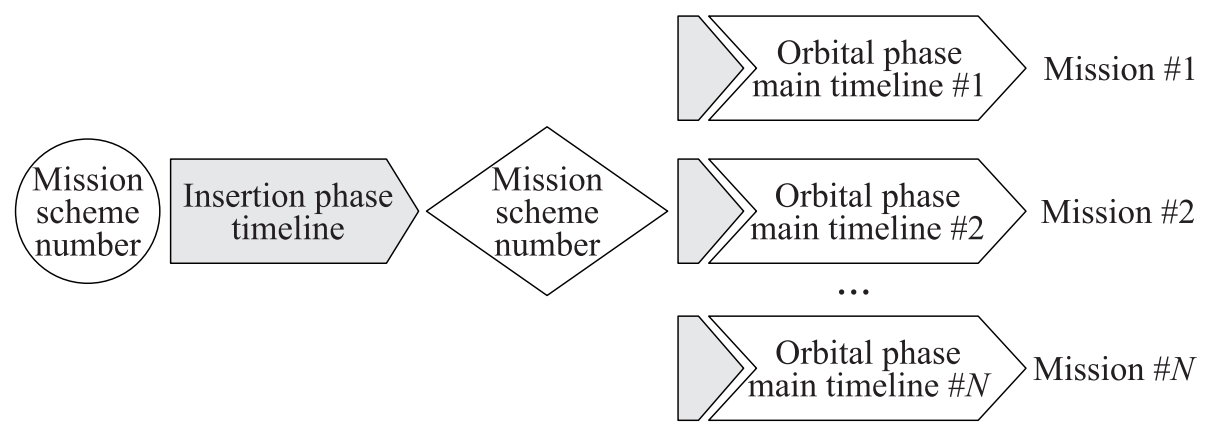

Figure 2 Main timeline identification

Orbital phase timeline includes all necessary legs of ME activation and long coasting phases of Homan's transfer for orbit change. This timeline is generated for every mission. Taking into account different combinations of such basic activities as ME ignitions, Long Coasting phases, etc., about ten different mission schemes can be determined. Each mission scheme is labeled by a unique identifier and has dedicated orbital phase timeline. The insertion phase timeline is unified for all mission schemes. The graphic interpretation of the main timeline identifications is given in Fig. 2.

After each ME activation, the orbital phase timeline has a set of Functional Commands to transfer to PL's separation timeline and then return back. The special parameter determines the number of PLs which have to be separated at that moment. A scheme of the transferring realization between main and additional timelines is given in Fig. 3.

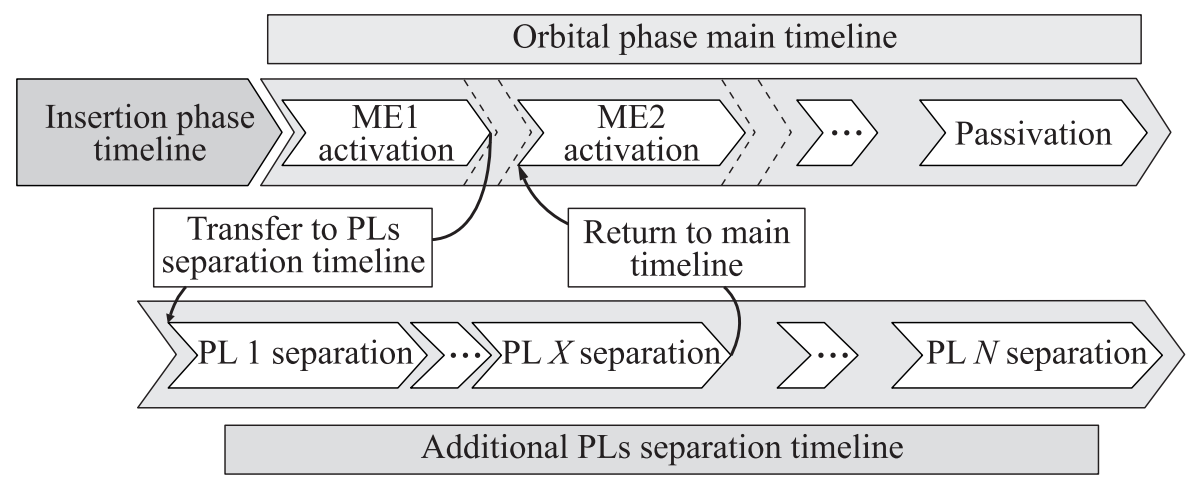

Figure 3 Scheme of the transferring realization between main and additional timelines 
Payload's separation timeline is an independent timeline that performs the separation of all PLs with all the required set of basic activities as PL pointing, spin, etc. A sequence of actions for each PL separation is specified at the mission preparation. The proposed method of additional timeline unites the advantages of the two approaches, described above, combining definiteness in the invariable phase of flight with flexibility at the orbital phase.

Thereby, multipayload logic received can be easy formalized, customized, and tested.

\subsection{Multipayload Logic on the Orbital Phase of Flight}

As a rule, the arranging of the orbital phase of flight is defined by the specific launcher characteristics (the possible number of $\mathrm{ME}$ activations). In general, the orbital phase of flight includes the following basic activities:

1. Long coasting phase before additional ME ignition.

The ME ignition for transferring to another orbit is realized once the required value of functional is reached. Heretofore, the launcher is moving on the orbit with ME cutting off. On this leg, it is possible to perform different activities defined in technical requirements to the Control system, launcher or PL, e.g., star correction of navigation system or spinning in the roll channel.

2. Attitude pointing maneuver before additional ME ignition.

Before ME ignition, it is necessary to implement the launcher orientation in the required direction. Parameters of the maneuver are determined based on information about the required (at the moment of PL separation) and actual orientation of launchers Body-fixed reference frames. Three-dimensional attitude pointing maneuver is more effective than a consecutive from the point of view of time costs, but has disadvantages from the positions of fuel economy, angular rate limitation, and the complexity of the realization. Often, maneuvering is realized by using low-thrust propulsion system. In each case, the decision about a type of maneuver is made based on the information about limitations, control elements effectiveness, and fuel consumption.

3. Additional ME ignition/cutoff. Transferring to other orbit.

Transferring the launcher to the other orbit is implemented by ME ignition at the defined point of orbit. The ME cutoff is realized when required value of functional, e. g., increment of velocity, is reached.

4. Long coasting phase before deorbiting.

Main engine ignition for deorbiting is realized when the required value of functional is reached. Heretofore, the launcher is moving on the orbit with ME cutting off. 
5. Attitude pointing maneuver before deorbiting.

Before ME ignition, it is necessary to implement the launcher orientation in the required direction.

6. Deorbiting (additional ME ignition).

Deorbiting is implemented by ME ignition at the defined point of the orbit. The ME cutoff is realized when the required value of functional, e.g., increment of velocity, is reached.

All these basic activities include a full set of the launcher flight operations which is necessary to realize in the orbital phase of flight.

All possible combinations of basic activities are analyzed on the preliminary design stage in order to ensure the realization of all possible mission schemes for a particular launcher. After that, each scheme is labeled by a unique identifier, which defines the structure of each main flight timeline (insertion phase and orbital phase timelines) and the sequence of functional commands and labels issue, including functional commands of transferring to PL's separation timeline.

Graphic interpretation of a hypothetical orbital phase timeline is presented in Fig. 4.
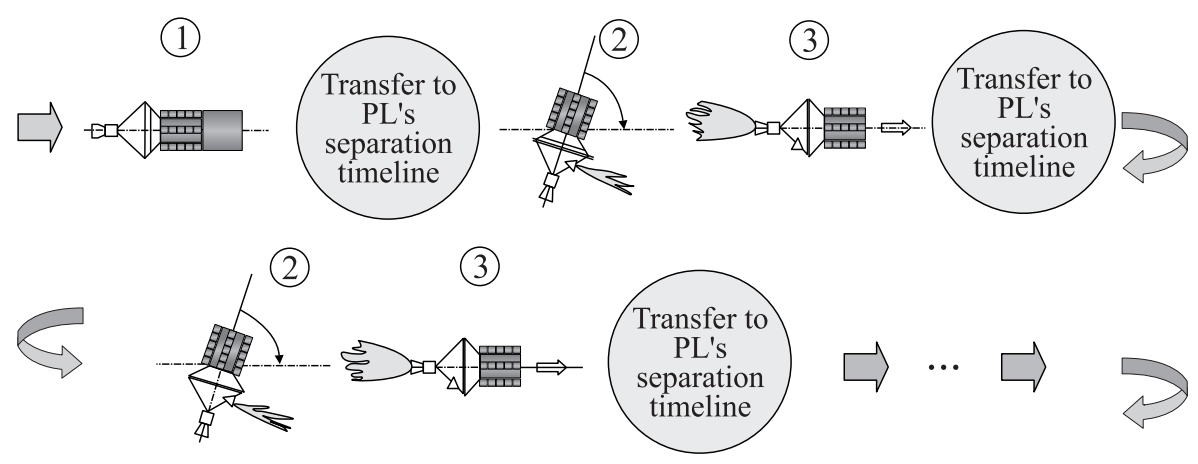

(4)

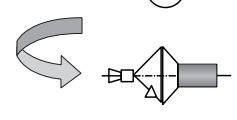

(5)

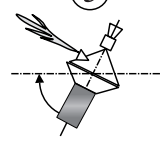

(6)
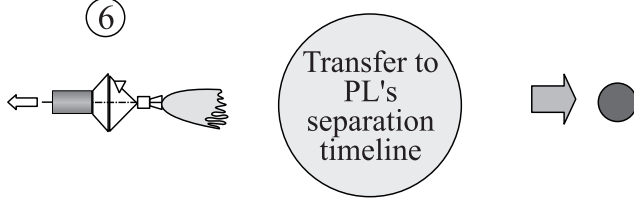

Figure 4 Orbital phase timelines 


\subsection{Multipayload Logic on the Payload's Separation Phase of Flight}

Arranging the PL's separation phase of flight is very important for ensuring the multipayload logic flexibility. Using the proposed method of additional timeline, it is necessary to provide the possibility of realization of any sequence of PL's separation. It is obtained by the maximum saturation of the PL's separation phase timeline by all the possible basic activities which have physical sense. In this case, the linkage of the basic activities with separation of each PL is ensured. This approach allows ensuring the usability of software customization in accordance with basic principles of synthesis described in subsection 2.4.

Below there are some basic activities.

1. Long coasting phase (start/finish of orbital phase of flight or separation leg of one of the PLs).

Main engine cutoff. Launcher was inserted on the required orbit. The actual attitude pointing of launcher Body-fixed reference frames (pitch, yaw, and roll) is determined. If necessary, some of the parameters (e.g., roll) could be realized by program values on the insertion phase of flight. It allows to ensure the maximum effectiveness of the next maneuvers, i. e., the actual and the required directions of launcher longitudinal axis are in one plane. This approach allows to decrease the fuel costs for maneuver implementation.

2. Attitude pointing maneuver before PL separation.

Main principles of the maneuver implementation are analogous to previous maneuvers.

3. Spin before PL separation.

Spin is implemented by using low-thrust propulsion system. Spin is rotation around axis $X$ of launcher Body-fixed reference frames with a defined angular rate. The spin is necessary because after separation, when the satellite is moving along orbit, it heats at the sunny side. The temperature drop between heated and cold sides could reach values that could influence the operability of the satellite. Spin, with constant angular rate, is used to avoid that. After the separation from the launcher, a satellite continues to rotate, which ensures acceptable temperature conditions.

4. Payload separation.

The command to PL separation is issued when the required conditions are reached, e.g., the time moment or the value of angular range. All limitations of attitude pointing and angular rate have to be implemented before separation. Payload separation is implemented in accordance with mission scheme. Microsatellites separation is implemented in accordance with general rules at 


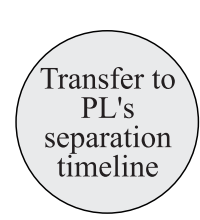

(1)

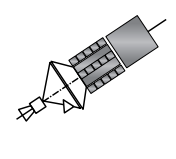

(4)
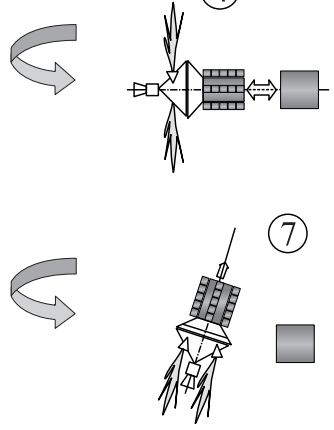

(7)
(2)

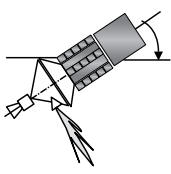

(5)
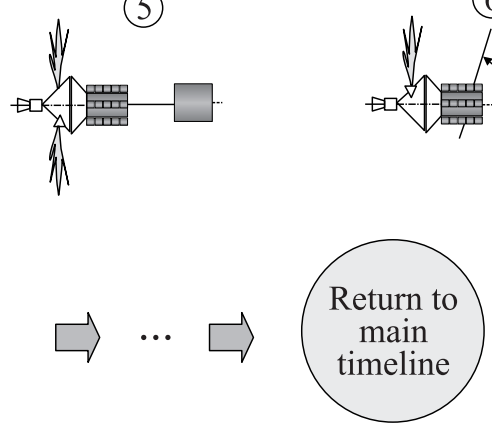

(3)

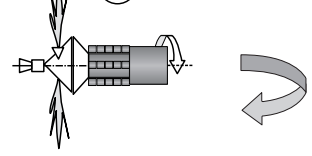

(6)

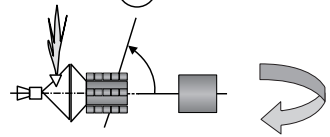

Figure 5 Payload separation phase timeline

each command as it is necessary, e.g., in pairs in direction perpendicular to the launcher longitudinal axis.

5. Despin after PL separation.

It is not necessary to continue launcher rotation after PL separation. Spin-off (angular rate in roll channel damping) is implemented by the way of applying of sign opposite spin torque. If necessary, the final value of the roll will ensure coincidence pitch plane and next attitude pointing maneuver plane.

6. Contamination/collision avoidance maneuver.

Launcher attitude pointing is transferred in safety direction after PL separation. Often, it has sign opposite to attitude pointing maneuver before PL separation. Main principles of the maneuver implementation are analogous to the previous maneuvers.

\section{Withdrawal.}

It is implemented by the way of ME or low-thrust propulsion system ignition. The launcher is withdrawn from PL to a safe distance.

Graphic interpretation of a hypothetical PL separation phase timeline is presented in Fig. 5.

Interaction between main flight timeline and additional PL's separation timeline is provided by the following algorithm: 
1. When the first target orbit was reached (first special functional command of the main flight timeline), transfer to start the PL's separation timeline is implemented.

2. All referred PLs are separated with implementation of all necessary basic activities in accordance with existing requirements for this target orbit.

3. Transfer to main flight timeline is implemented after fulfillment of all scheduled basic activities. Transfer is realized into the interval between the last special functional command and the next functional command of the main flight timeline according to the mission scheme.

4. When the next target orbit is reached, all operations repeat, with one difference - transfer to PL's separation phase timeline is implemented on the leg relevant to the number of PLs that have to be separated.

Thereby, it is ensured that there is no dependence between flight timeline and the number of PLs separated, as there are no limitations on the length of PL's separation phase timeline.

\section{CONCLUDING REMARKS}

During multipayload injection conception development, the following scientific and methodological problems were formulated and solved:

- clear interpretation of multipayload injection logic concepts was proposed;

- set of basic activities was specified;

- main principles of flight timeline synthesis were proposed and justified;

- method of additional PL's separation timeline was designed; and

- method provides transfer from the main timeline to additional one and return.

Implementation of the proposed approach allows to obtain the following advantages:

- each developer of the launcher and Control system will use unified terminology;

- each developer of the launcher and Control system will use unified set of basic activities; 
- the process of synthesis of timeline for each existing or perspective launcher will be unified; and

- the developed algorithms of the Control system will be adequate to any multipayload injection scheme.

The advantages mentioned allow to standardize the process of design in cooperation, to increase its effectiveness, and to decrease time costs for coordination, correction, and testing onboard SW for a particular mission. 